LIVER

\title{
Asymptomatic primary biliary cirrhosis: clinical features, prognosis, and symptom progression in a large population based cohort
}

\author{
M I Prince, A Chetwynd, W L Craig, J V Metcalf, O F W James
}

Gut 2004;53:865-870. doi: 10.1136/gut.2003.023937

Background: Many patients with primary biliary cirrhosis $(\mathrm{PBC})$ are asymptomatic at the time of diagnosis. However, because most studies of asymptomatic PBC have been small and from tertiary centres, asymptomatic $\mathrm{PBC}$ remains poorly characterised.

Aims: To describe the features and progression of initially asymptomatic PBC patients.

Methods: Follow up by interview and note review of a large geographically and temporally defined cohort of patients with $\mathrm{PBC}$, collected by multiple methods.

See end of article for authors' affiliations ....................

Correspondence to: Dr Martin Prince, Newcastle University Medical School, Framlington PI, Newcastle NE2 $4 \mathrm{HH}$, UK; martin. prince@ncl.ac.uk

Accepted for publication 1 December 2003
Results: Of a total of 770 patients, 469 (61\%) were asymptomatic at diagnosis. These patients had biochemically and histologically less advanced disease than initially symptomatic patients. Median survival was similar in both groups (9.6 $\vee 8.0$ years, respectively) possibly due to excess of non-liver related deaths in asymptomatic patients ( $31 \%$ v $57 \%$ of deaths related to liver disease). Survival in initially asymptomatic patients was not affected by subsequent symptom development. By the end of follow up, $20 \%$ of initially asymptomatic patients had died of liver disease or required liver transplantation. The majority of initially asymptomatic patients developed symptoms of liver disease if they were followed up for long enough (Kaplan-Meier estimate of proportion developing symptoms: 50\% after five years, $95 \%$ after 20 years). However, $45 \%$ of patients remained asymptomatic at the time of death.

Conclusions: Although asymptomatic PBC is less severe at diagnosis than symptomatic disease, it is not associated with a better prognosis, possibly due to an increase in non-hepatic deaths. The reasons for this are unclear but may reflect confounding by other risk factors or surveillance bias. These findings have important implications for future treatment strategies.
$\mathrm{R}$ ecent estimates suggest that there are 12 000-15 000 patients with primary biliary cirrhosis (PBC) in the UK ${ }^{1}$ and 40000 in the USA. ${ }^{2}$ PBC was initially described as a universally severe condition, presenting with pruritus and jaundice and usually progressing rapidly to liver failure in less than three years. ${ }^{3-5}$ The discovery of antimitochondrial antibodies (AMA), ${ }^{6}$ and their subsequent inclusion in "routine" autoantibody profiles, has led to PBC being diagnosed more frequently and often at an earlier stage in the disease process. Fox et al were the first to describe PBC presenting with no symptoms attributable to liver disease ("asymptomatic PBC") in 1973.7 Subsequent studies redefined the spectrum of disease phenotypes to include a high proportion of such asymptomatic patients. ${ }^{8-10}$ However, the clinical course, particularly with respect to prognosis and symptom development, of asymptomatic PBC remains relatively poorly described with case series being small (less than 91 initially asymptomatic patients) and largely based in tertiary referral centres. ${ }^{7-20}$

By the early 1990s, the consensus of case series of initially asymptomatic patients was that: (i) after about six years, two thirds of initially asymptomatic patients would develop symptoms; (ii) once symptoms developed, prognosis was as for the generality of PBC patients; and (iii) overall mortality in initially asymptomatic patients was higher than age matched populations but only after 10 or more years, and lower than in initially symptomatic patients.

As up to $60 \%$ of PBC patients are now asymptomatic at diagnosis, it is timely to re-examine the outcome of asymptomatic PBC in a large unselected cohort of patients to gain a clearer idea of its true early natural history. This has become particularly important in helping to make treatment decisions and to inform the debate about the need to treat patients with, for example, ursodeoxycholic acid (UDCA) while they remain asymptomatic of their liver disease. ${ }^{21}$ We have recently examined the overall natural history of an established unselected cohort of 770 patients, defined between 1987 and 1994. ${ }^{22}$ However, we presented little specific data in respect of the 469 patients who were asymptomatic of liver disease at diagnosis. The present report examines the clinical features, symptom progression, and outcomes in these initially asymptomatic patients.

\section{METHODS}

The methods used to identify the Northeast England cohort of patients with PBC have previously been described. ${ }^{1}$ This cohort included all incident and prevalent cases of definite or probable PBC alive between 1 January 1987 and 31 December 1994 within a geographically defined area of Northeast England. Multiple case finding methods were employed to ensure completeness of the cohort.

Definite PBC was defined as all three of: abnormal liver function tests, positive AMA, and compatible liver histology. Probable PBC was defined as any two of these. Data here includes both probable and definite disease. Date of diagnosis was defined as the first date when two of these diagnostic criteria were found to be present.

Abbreviations: $\mathrm{PBC}$, primary biliary cirrhosis; $\mathrm{AMA}$, antimitochondrial antibodies; UDCA, ursodeoxycholic acid; SMR, standardised mortality ratio 
Table 1 Features at diagnosis of asymptomatic and asymptomatic patients

\begin{tabular}{|c|c|c|c|}
\hline & $\begin{array}{l}\text { Initially asymptomatic } \\
\text { patients ( } n=469 \text { ) }\end{array}$ & $\begin{array}{l}\text { Initially symptomatic } \\
\text { patients }(n=301)\end{array}$ & p Value \\
\hline Median age (y) & 63.1 & 61.8 & 0.10 \\
\hline No female (\%) & $412(87 \%)$ & $282(93 \%)$ & 0.008 \\
\hline \multicolumn{4}{|l|}{ Autoantibody status* } \\
\hline AMA positive ( $>1$ in 40 ) & $422(90.3 \%)$ & $268(89.3)$ & 0.73 \\
\hline ANF positive & $68(14.6 \%)$ & $37(12.3 \%)$ & 0.44 \\
\hline SMA positive & $24(5.1 \%)$ & $17(5.6 \%)$ & 0.88 \\
\hline AMA titre $>1$ in 60 & $136(29.1 \%)$ & $102(34.0 \%)$ & 0.06 \\
\hline \multicolumn{4}{|l|}{ LFTs at diagnosis $†$} \\
\hline Alkaline phosphatase & $2.09(1.30-4.20)$ & $2.47(1.42-5.94)$ & 0.005 \\
\hline Alanine transaminase & 1.12 (0.71-2.00) & 1.48 (0.88-2.67) & 0.001 \\
\hline Bilirubin $(\mu \mathrm{mol} / \mathrm{l})$ & $8.9(7.0-15.0)$ & $10.9(7.0-23.9)$ & $<0.001$ \\
\hline Prothrombin ratio & $1.0(0.91-1.00)$ & $1.0(0.92-1.0)$ & 0.91 \\
\hline \multicolumn{4}{|l|}{ Histology at diagnosis $\ddagger$} \\
\hline Precirrhotic & $146(84 \%)$ & 107 (68\%) & \\
\hline Cirrhotic & $28(16 \%)$ & $51(32 \%)$ & $<0.001$ \\
\hline
\end{tabular}

Patients were defined as initially asymptomatic if they did not have symptoms attributable to their liver disease at the date of diagnosis. The presence of symptoms at diagnosis was determined from medical records and by initial interview of patients by the investigators. Symptoms of PBC were defined as pruritus, persistent fatigue (lasting greater than three months in the absence of other identifiable causes such as anaemia or hypothyroidism), persistent right hypochondrial pain in the absence of another cause such as cholelithiasis, overt jaundice, bleeding oesophageal varices, ascites, or hepatic encephalopathy. Liver failure was defined as any of the last three symptoms.

Patients were followed up by regular interview and by examination of their medical records until 1 January 2000 or death. Deaths were defined as being "liver related" after review of the notes if they were related to a complication of liver disease (including hepatocellular carcinoma). Patient deaths were noted by examination of all clinical records and by logging all patient details with the Office of National Statistics.

Results were analysed using $\chi^{2}$ and Mann-Whitney U tests. Survival data were analysed by the Kaplan-Meier method and Cox proportional hazards regression. Survival times were adjusted in the Cox proportional hazards regression to take account of the presence of cases that were prevalent at the outset of the study using the methods described by Kieding. ${ }^{23}$ The presence of these cases could otherwise introduce bias

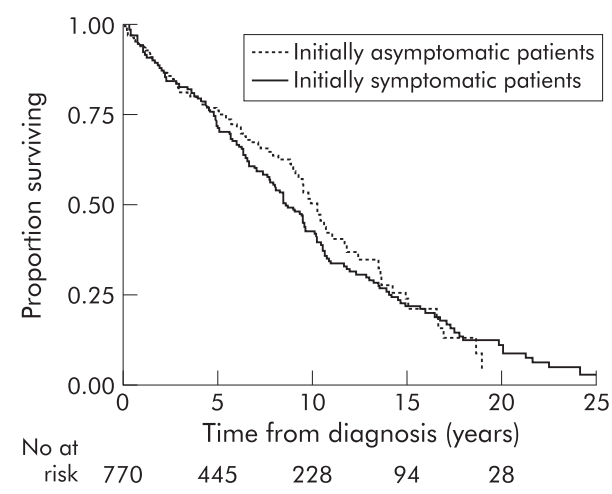

Figure 1 Kaplan-Meier estimates of patient survival from the time of diagnosis in initially asymptomatic and symptomatic patients. into survival estimates as the survival times of prevalent cases are positively biased compared with those in the underlying population. The database complied with data protection legislation and local ethics committees approved this study.

Liver function tests were reported as the ratio of the test result to the upper limit of normal for the laboratory performing the test to account for tests being undertaken in multiple hospitals with differing normal ranges and units of measurement. For survival data, patient information was censored at 1 January 2000 for living patients. For symptom data, patient information was censored at the last clinic visit where symptoms may have been recorded, or death if the patient remained asymptomatic from their liver disease at this point. The records of patients who underwent liver transplantation were censored at the time of surgery. Indirect standardised mortality ratios (SMRs) were calculated at 1 January 1999 (the last date for which regional population data were available). SMRs were calculated using five year age-sex bands. General population data were obtained from mortality statistics for the Northern Regional Health Authority (which very largely corresponds to the study area). Indirect standardisation (as compared with direct) avoids large error in estimates due to very small numbers of deaths observed in individual bands, but means that individual SMRs cannot be compared with each other.

Table 2 Primary cause of death according to death certificate data

\begin{tabular}{|c|c|c|}
\hline $\begin{array}{l}\text { Cause of death as recorded on } \\
\text { death certificate }\end{array}$ & $\begin{array}{l}\text { Initially } \\
\text { asymptomatic } \\
\text { patients (n (\%)) }\end{array}$ & $\begin{array}{l}\text { Initially } \\
\text { symptomatic } \\
\text { patients (n (\%)) }\end{array}$ \\
\hline Non-hepatic malignancies & $47(19.0)$ & $16(9.4)$ \\
\hline Hepatocellular carcinoma & $5(2.0)$ & $9(5.3)$ \\
\hline Ischaemic heart disease & $37(14.9)$ & $10(5.9)$ \\
\hline Cerebrovascular disease & $17(6.9)$ & $12(7.1)$ \\
\hline Peripheral vascular disease & $3(1.2)$ & $0(0)$ \\
\hline Other cardiac disorders & $6(2.4)$ & $6(3.5)$ \\
\hline $\begin{array}{l}\text { Non-malignant respiratory disease } \\
\text { (including pneumonia) }\end{array}$ & $51(20.6)$ & $32(18.8)$ \\
\hline Liver failure or variceal bleeding & $63(25.4)$ & $75(44.1)$ \\
\hline $\begin{array}{l}\text { Non-malignant gastrointestinal } \\
\text { disease }\end{array}$ & $5(2.0)$ & $2(1.2)$ \\
\hline Miscellaneous & $14(5.6)$ & $8(4.7)$ \\
\hline
\end{tabular}




\section{RESULTS}

Of 770 patients with PBC (472 definite, 298 probable) in the Northeast England cohort, 469 were asymptomatic from PBC at the time of diagnosis $(60.9 \%$ (95\% confidence interval $57.4-64.4 \%)$ ).

Data to allow calculation of survival corrected for the effect of prevalent cases were available for 765 patients (99\%). Initial interview to determine symptoms at the time of diagnosis was possible for 354 of 469 (75\%) initially asymptomatic patients. Initial medical record review was possible for all patients. Full clinical symptom follow up data by interview and regular case note review were possible for $422(90 \%)$ initially asymptomatic patients.

Median follow up for mortality data was 7.4 years (range $0-28$ ). Median follow up for symptom data was 7.3 years (range 0-28).

The clinical features of initially asymptomatic patients are shown in table 1. Median age at diagnosis was similar in initially asymptomatic and symptomatic patients $(63.1 \mathrm{v}$ 61.8 years). A total of $260(55.4 \%)$ asymptomatic patients were aged less than 65 years at the time of diagnosis. The severity of $\mathrm{PBC}$, whether assessed through serum liver function tests or liver histology, was milder in patients with initially asymptomatic disease than initially symptomatic patients. However, relatively few patients in either group had markedly abnormal liver function tests at diagnosis (proportion of patients with bilirubin greater than twice the upper limit of normal, $8 \%$ asymptomatic $v 17 \%$ symptomatic).

A total of $248(52.8 \%)$ initially asymptomatic patients died during follow up. As shown in fig 1 , although median survival time from diagnosis was slightly longer in asymptomatic than in symptomatic patients (9.6 years (95\% confidence limits $8.6-10.5) \quad v \quad 8.0$ years $(6.6-10.0))$, this difference was not statistically significant $(p=0.212)$. This result was unchanged in multivariate survival analysis, including prognostic variables such as age, sex, and initial liver function tests. Censoring patients at the point of transplantation had a small effect on overall survival estimates (approximately one year in all estimates) and did not alter the relative survival of symptomatic and asymptomatic patients.

The primary cause of death, as recorded on patients' death certificates, is shown in table 2. Twenty four deaths (20 in initially asymptomatic patients) were due to lung cancer. Patient death was due to atheromatous vascular disease in 57 ( $24 \%$ of deaths) initially asymptomatic patients. Twenty two deaths were classified as being due to liver disease following case note review but did not have liver disease listed as the

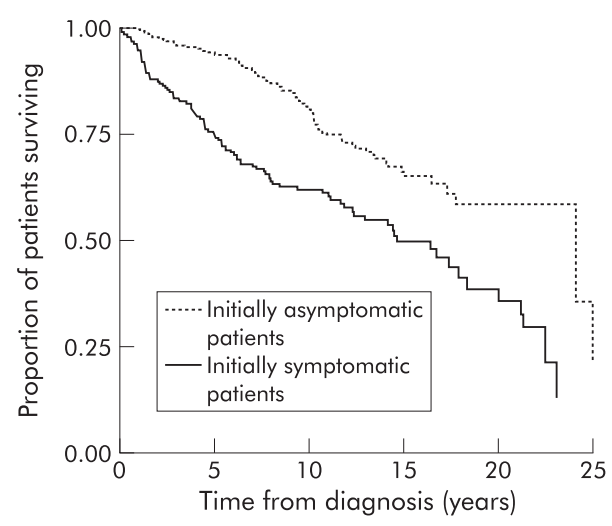

Figure 2 Kaplan-Meier estimates of patient mortality due to liver disease from the time of diagnosis in initially asymptomatic and symptomatic patients.

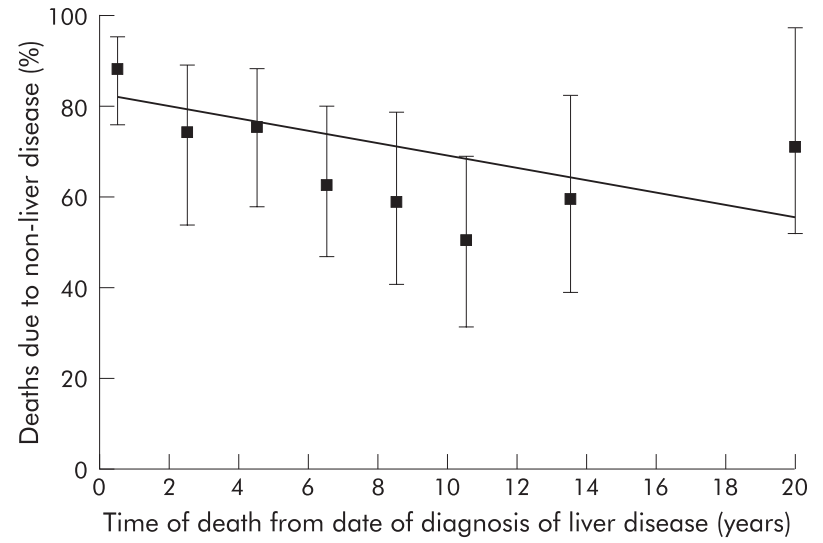

Figure 3 Proportion of deaths (with $95 \%$ confidence interval) attributable to liver disease in initially asymptomatic patients at increasing time from diagnosis.

Table 3 Kaplan-Meier estimates of the proportion of initially asymptomatic patients developing symptoms of liver disease during the first 10 years of follow up

\begin{tabular}{lccc}
\hline & \multicolumn{3}{l}{$\begin{array}{l}\text { Proportion of patients developing } \\
\text { symptoms (\%) }\end{array}$} \\
\cline { 2 - 4 } Symptoms & \multicolumn{3}{c}{ Time since diagnosis } \\
\cline { 2 - 4 } & $\mathbf{1}$ year & $\mathbf{5}$ years & $\mathbf{1 0}$ years \\
\hline Itch & 15.5 & 30.8 & 46.5 \\
Fatigue & 13.1 & 29.1 & 45.8 \\
Hypochondrial pain & 4.0 & 8.1 & 14.4 \\
Bone pain & 3.7 & 7.8 & 15.4 \\
Ascites & 1.7 & 8.1 & 16.7 \\
Bleeding varices & 1.0 & 3.4 & 9.2 \\
Hepatic encephalopathy & 1.3 & 3.7 & 8.7 \\
Jaundice & 2.8 & 9.0 & 17.0 \\
Any symptom of PBC & 26.4 & 50.7 & 71.3 \\
Liver failure & 3.0 & 12.2 & 23.6 \\
\hline
\end{tabular}

primary cause of death on their death certificate. These deaths have therefore been ascribed to liver disease.

The proportion of deaths that were related to liver disease was lower in initially asymptomatic than symptomatic patients ( 77 deaths (31\% of deaths) $v 97$ deaths $(57 \%)$ were related to liver disease respectively; $p=0.004$ ). As shown in fig 2, mortality due to liver disease was lower in initially asymptomatic than symptomatic patients (median survival with non-liver deaths excluded 14.6 years (12.6-20.1) $v$ 24.1 years (17.7 to infinity)). As shown in fig 3, the proportion of deaths in asymptomatic patients due to nonliver related causes decreased with over time between diagnosis and death $\left(\chi^{2}\right.$ for linear trend $=7.53$ ( $\left.1 \mathrm{df}\right)$, $\mathrm{p}=0.006 ; \chi^{2}$ for residual $\left.=10.2(6 \mathrm{df}) ; \mathrm{p}>0.05\right)$.

The indirect SMR for asymptomatic patients was 2.64 (95\% confidence limits 2.30-3.02) and was 1.86 (1.57-2.18) excluding liver related deaths. Similar SMRs in initially symptomatic patients were $3.22(2.74-3.77)$ and 1.49 (1.16$1.87)$, respectively.

A total of $255(60.5 \%)$ of 422 initially asymptomatic patients for whom follow up data were available developed symptoms of liver disease during follow up. Table 3 gives the Kaplan-Meier estimate of the proportion of initially asymptomatic patients developing each symptom of liver disease 1, 5, and 10 years after the date of possible diagnosis. Approximately half $(51 \%)$ of initially asymptomatic patients 


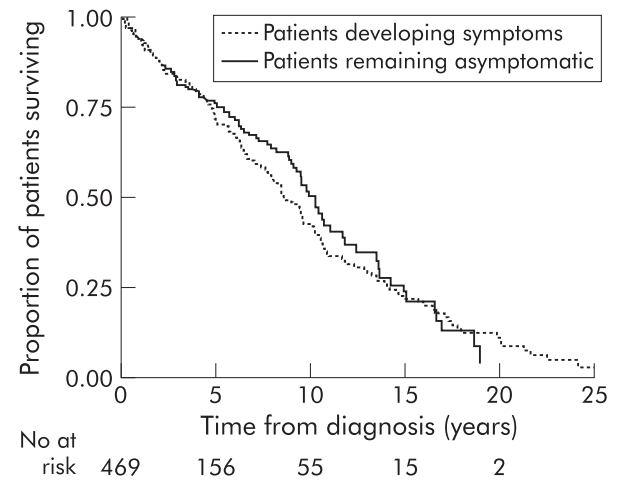

Figure 4 Kaplan-Meier estimates comparing patient survival in initially asymptomatic patients before and after symptom development.

had developed symptoms of PBC within five years of diagnosis and 95\% after 20 years. However, 112 (45\%) of 248 initially asymptomatic patients who died during follow up, died prior to the development of liver related symptoms and of causes unrelated to their liver disease.

The effect on survival of symptom development in initially asymptomatic patients was analysed using a time dependent Cox proportional hazards model. Data were available to perform this calculation for $413(98 \%)$ patients. All clinical features, except liver histology at diagnosis, of these 413 initially asymptomatic patients were used in a time independent model of hazard rate. The development of a first symptom of PBC was added into this model in a time dependent fashion. In the adjusted model, symptom development was associated with a small increase in hazard rate which was not statistically significant $(p=0.34)$, as shown in fig 4. Repeat modelling, including liver histology, did not change this finding.

Nineteen $(7.3 \%$ of patients aged less than 65 years at diagnosis) initially asymptomatic patients underwent liver transplantation during the period of follow up. A total of 133 $(31.5 \%)$ initially asymptomatic patients received UDCA, of whom 99 (74.4\%) had developed symptoms prior to starting therapy. Thus only 34 patients had received UDCA before symptom development. The median dose of UDCA was $450 \mathrm{mg} /$ day.

\section{DISCUSSION}

As discussed above, with wider diagnosis the recognised phenotype of PBC has changed from a universally severe disorder $^{35}$ to a heterogeneous condition that is often asymptomatic when initially diagnosed. Six groups have subsequently reported their experience of asymptomatic PBC. ${ }^{11}{ }^{13-19}$ These comprised between $13 \%{ }^{16}$ and $70 \%{ }^{15}$ of PBC patients in their case series.

Direct comparison between these series is difficult due to differences in patient populations (largely derived from tertiary referral centres and therefore possibly subject to Berkson's bias $)^{9111^{13-15}}$ and varying definitions of the symptoms of PBC (and hence asymptomatic PBC). For example, Springer et al included 18 patients with persistent fatigue among 91 "asymptomatic" patients ${ }^{19}$ and Roll et al five patients with overt jaundice among 37 "asymptomatic" patients. ${ }^{16}$ Finally, these series were relatively small, ranging in size from $17^{9}$ to $91^{19}$ asymptomatic patients.

This is the first report of strictly defined asymptomatic PBC in a cohort that was relatively free of selection and referral biases, and with a strict definition of the date of diagnosis. In this large group, slightly more than half $(61 \%)$ of all patients were asymptomatic at the time that diagnosis was possible.
Three previous studies have compared the laboratory features at diagnosis of asymptomatic and symptomatic patients. Roll et al found that patients with asymptomatic PBC were more likely to have a lower AMA titre and less advanced histological fibrosis than symptomatic patients. ${ }^{16}$ Nyberg and Loof confirmed that asymptomatic PBC was associated with histologically less advanced disease. ${ }^{15}$ Uddenfeldt and Danielsson reported that asymptomatic patients had lower alkaline phosphatase, bilirubin, and transaminase levels. ${ }^{20}$ Our series confirmed all of these except the autoantibody differences.

Five surveys have previously reported on the survival of initially asymptomatic PBC but none of these studies accounted for the effect of prevalent cases on estimates of survival. Nyberg and Loof followed up 56 initially asymptomatic patients for a mean of 9.5 years. ${ }^{15}$ Median survival ( 12.7 years) was better than in symptomatic patients (8.0 years) but worse than expected for the general population, although it took 12 years for this to become statistically significant. The Yale liver unit reported the survival of 37 initially asymptomatic patients. These patients had better median survival than initially symptomatic patients ( 16 years $v 7.5$ years) but worse than the general population, which became apparent after 11 years of follow up. ${ }^{14}{ }^{17}$ Balasubramaniam et al and Springer and colleagues ${ }^{11} 19$ reported that patients with asymptomatic PBC had decreased survival compared with the general population (median survival 9.5 and 14 years, respectively) although they did not compare this with survival in initially symptomatic PBC patients. In contrast, Uddenfeldt and Danielsson ${ }^{20}$ found that patients with asymptomatic PBC had similar survival to the normal population, which again was better than in symptomatic patients. However, all five studies were based on very few (all less than 18) patient deaths. (The total number of deaths in all five studies combined was just 47 compared with 248 in the present study.)

Thus until now the consensus has been that survival is better in initially asymptomatic than symptomatic patients. Our study reports survival over a longer period and with a very much larger number of patient deaths than previous series, and did not confirm this finding. We confirmed that asymptomatic PBC has a reduced survival compared with the general population (with an SMR over two and a half times as high as expected). ${ }^{11}{ }^{13-15}$ We did not however find that initial symptom status affected subsequent survival, despite asymptomatic patients having lower median bilirubin and less cirrhosis on biopsy than symptomatic patients at diagnosis. Two possible reasons for this are firstly, that although the median bilirubin level was higher in symptomatic patients, relatively few patients had markedly elevated bilirubin levels. Secondly, any improvement in liver related survival in initially asymptomatic patients might have been outweighed by an excess of non-hepatic deaths, particularly in the asymptomatic group.

This is first study to examine non-liver related mortality in asymptomatic PBC. After excluding liver deaths, the mortality rate remained nearly twice that expected. There are several possible reasons for this excess. Firstly, asymptomatic PBC may have been diagnosed during investigation of unrelated conditions and the excess mortality was due to this comorbidity (surveillance bias). The excess of non-liver deaths early after diagnosis supports this possibility. Secondly, two case control studies have previously found an excess of smoking in patients with $\mathrm{PBC}^{24} 25$ and the excess mortality observed here may reflect non-hepatic smoking related adverse events. However, the rates of atheromatous disease and lung cancer, as recorded on death certificates, were broadly similar to those in the local population, suggesting that, if present, this effect is relatively small. 
Three previous studies have failed to find an excess of mortality due to coronary artery disease in patients with PBC. ${ }^{26-28}$ Thirdly, the excess mortality may reflect an association between PBC and other diseases. Patel et al found an excess of autoimmune disease in patients with $\mathrm{PBC}$ compared with their siblings and friends. ${ }^{25} \mathrm{PBC}$ has been reported to be associated with an excess of non-hepatic malignancy. ${ }^{29-31}$ A previous study of this cohort followed for a median of 5.4 years found a small but statistically significant excess of non-hepatic cancers when the effect of increased surveillance was accounted for (standardised incidence ratio 1.7 (95\% confidence limits $1.3-2.2)){ }^{32}$

Our study is the first to examine the effect of symptom development on patient survival in a time dependent manner. Symptom development did not significantly $(p=0.34)$ affect patient survival. The lack of a marked effect of symptom development on survival is perhaps to be expected as, in contrast with the above studies, we did not find that survival was different in initially symptomatic and asymptomatic patients. Two previous studies suggested that initially asymptomatic disease had a better prognosis than symptomatic disease only until the development of symptoms. ${ }^{13}{ }^{14}$ However, these studies reported on very small numbers of deaths in patients who developed symptoms ( 15 deaths and two deaths, respectively). Furthermore, they used analysis methods that did not account for the time delay between date of diagnosis and onset of symptoms.

This new perspective may also change attitudes as to when to introduce treatment for patients with asymptomatic PBC. As prognosis is broadly similar to symptomatic patients, it may be argued that, as in the case of asymptomatic systemic hypertension for example, treatment of patients with asymptomatic PBC should be started where possible at the date of diagnosis.

This is the first study to examine the time course of symptom development in initially asymptomatic patients. Previous studies have reported symptom development in 21 $(60 \%)$ of $35,{ }^{15} 24(67 \%)$ of $36,{ }^{14} 33(36 \%)$ of 91 , and $33(89 \%)$ of $37^{11}$ initially asymptomatic patients followed for varying periods. However, none of these studies reported the time course of symptom progression. Kaplan-Meier analysis in our cohort clearly shows that the asymptomatic phase of PBC can be regarded as a temporary phenomenon. If patients survive for a long enough period, virtually all will develop one or more symptoms of PBC (95\% after 20 years). However, we also found that many patients died before developing symptoms of PBC (45\% of deaths occurred in patients who were still asymptomatic), reflecting the age of patients at diagnosis and variation in the progress of disease. The median age at diagnosis in this study (62 years) was 5-10 years higher than in most previous studies-perhaps reflecting referral patterns to tertiary referral centres. This greater age may also explain our higher proportion of nonliver deaths.

Only a small proportion of asymptomatic patients received therapy with UDCA prior to symptom development. The median UDCA dose (450 mg) used is now thought to be subtherapeutic, ${ }^{33}$ although it is controversial as to whether UDCA affects the prognosis of PBC. ${ }^{34}{ }^{35}$ The pattern of UDCA usage may reflect the time period from which patients were drawn and varying prescribing habits among gastroenterologists in the study region.

In conclusion, $20 \%$ of initially asymptomatic patients died of liver disease or required transplantation. Furthermore, overall prognosis did not differ from patients presenting with symptoms. These findings contrast with the previous assumption that asymptomatic PBC represents a largely mild form of disease and may have profound implications in future considerations of treatment of disease.

\section{ACKNOWLEDGEMENTS}

The study was supported by the Wellcome Trust (JVM) and the Northern and Yorkshire Regional Health Authority and Medical Research Council (MP).

We are grateful to all of the gastroenterologists and other physicians in Northeast England for their continued support and assistance throughout this study.

\section{Authors' affiliations}

M I Prince, A Chetwynd, W L Craig, J V Metcalf, O F W James, Newcastle University Medical School, Newcastle, UK

\section{REFERENCES}

1 James OF, Bhopal R, Howel D, et al. Primary biliary cirrhosis once rare, now common in the United Kingdom? Hepatology 1999;30:390-4.

2 Kim WR, Lindor KD, Locke GR 3rd, et al. pidemiology and natural history of primary biliary cirrhosis in a US community. Gastroenterology 2000; 1 19:1631-6.

3 Sherlock S. Primary biliary cirrhosis (chronic intrahepatic obstructive jaundice). Gastroenterology 1959;37:574-86.

4 Addison T, Gull W. On a certain affliction of the skin-vitiligoides-a planus tuberosa. Guys Hospital Reports 1857;7:268.

5 Ahrens E, Payne M, Kunkel $\mathrm{H}$, et al. Primary biliary cirrhosis. Medicine 1950;29:299-366.

6 Walker J, Doniach D, Roitt I, et al. Serological tests in diagnosis of primary biliary cirrhosis. Lancet 1965;i:827-33.

7 Fox RA, Scheuer PJ, Sherlock S. Asymptomatic PBC. Gut 1973;14:444-7.

8 James $\mathrm{O}$, Macklon AF, Watson AJ. Primary biliary cirrhosis - a revised clinical spectrum. Lancet 1981;1:1278-81.

9 Crowe J, Christensen E, Doniach D, et al. Early features of primary biliary cirrhosis: an analysis of 85 patients. Am J Gastroenterol 1985;80:466-8.

10 Long RG, Schever PJ, Sherlock S. Presentation and course of asymptomatic primary biliary cirrhosis. Gastroenterology 1977:72:1204-7.

11 Balasubramaniam K, Grambsch PM, Wiesner RH, et al. Diminished survival in asymptomatic primary biliary cirrhosis. A prospective study. Gastroenterology 1990;98:1567-71.

12 Eriksson S, Lindgren S. The prevalence and clinical spectrum of primary biliary cirrhosis in a defined population. Scand J Gastroenterol 1984; 19:971-6.

13 Mitchison HC, Lucey MR, Kelly PJ, et al. Symptom development and prognosis in primary biliary cirrhosis: a study in two centres. Gastroenterology 1990;99:778-84.

14 Mahl TC, Shockcor W, Boyer JL. Primary biliary cirrhosis: survival of a large cohort of symptomatic and asymptomatic patients followed for 24 years. $J$ Hepatol 1994;20:707-13.

15 Nyberg A, Loof L. Primary biliary cirrhosis: clinical features and outcome, with special reference to asymptomatic disease. Scand J Gastroenterol 1989;24:57-64.

16 Roll J, Boyer JL, Barry D, et al. The prognostic importance of clinical and histologic features in asymptomatic and symptomatic primary biliary cirrhosis. N Engl J Med 1983;308:1-7.

17 Beswick DR, Klatskin G, Boyer JL. Asymptomatic primary biliary cirrhosis. A progress report on long-term follow-up and natural history. Gastroenterology 1985;89:267-71.

18 Witt-Sullivan H, Heathcote J, Cauch K, et al. The demography of primary biliary cirrhosis in Ontario, Canada. Hepatology 1990;12:98-105.

19 Springer J, Cauch-Dudek K, O'Rourke K, et al. Asymptomatic primary biliary cirrhosis: a study of its natural history and prognosis. Am J Gastroenterol 1999:94:47-53.

20 Uddenfeldt $P$, Danielsson A. Primary biliary cirrhosis: survival of a cohort followed for 10 years. J Intern Med 2000;248:292-8.

21 Neuberger J. URSO-panacea or placebo? Hepatology 2000;31:1027-8.

22 Prince M, Chetwynd A, Craig W, et al. Patient survival and symptom progression in a large geographically based cohort of patients with PBC: Follow up for up to 28 years. Gastroenterology 2002; 123:1044-51.

23 Goel P, Klein J, eds. Independent delayed entry. Proceedings of the NATO advanced statistics workshop on survival analysis and related topics. Columbus, Ohio: Kluwer Academic Publishers, 1991.

24 Howel D, Fischbacher C, Bhopal R, et al. An exploratory population-based case-control study of primary biliary cirrhosis. Hepatology 2000:31:1055-60.

25 Patal A, Gold E, Davis C, et al. A epidemiological analysis of risk factors in PBC. Hepatology 1999;30(suppl 2):471A.

26 Crippin JS, Lindor KD, Jorgensen R, et al. Hypercholesterolemia and atherosclerosis in primary biliary cirrhosis: what is the risk? Hepatology 1992;15:858-62.

27 Van Dam GM, Gips CH. Primary biliary cirrhosis in The Netherlands. An analysis of associated diseases, cardiovascular risk, and malignancies on the basis of mortality figures. Scand J Gastroenterol 1997;32:77-83.

28 Longo $M$, Crosignani $A$, Battezzati $P$, et al. Hyperlipidaemic state and cardiovascular risk in primary biliary cirrhosis. Gut 2002;51:265-9.

29 Loof L, Adami HO, Sparen P, et al. Cancer risk in primary biliary cirrhosis: a population-based study from Sweden. Hepatology 1994;20(1 Pt 1):101-4. 
30 Niihawan PK, Therneau TM, Dickson ER, et al. Incidence of cancer in primary biliary cirrhosis: the Mayo experience. Hepatology 1999;29:1396-8.

31 Wolke AM, Schaffner F, Kapelman B, et al. Malignancy in primary biliary cirrhosis. High incidence of breast cancer in affected women. Am J Med, 1984;76:1075-8.

32 Howel D, Metcalf JV, Gray J, et al. Cancer risk in primary biliary cirrhosis: a study in northern England. Gut 1999;45:756-60.
33 Eriksson LS, Olsson R, Glauman $\mathrm{H}$, et al. Ursodeoxycholic acid treatment in patients with primary biliary cirrhosis. A Swedish multicentre, double-blind, randomized controlled study. Scand J Gastroenterol 1997;32:179-86.

34 Gluud C, Christensen E. Ursodeoxycholic acid for primary biliary cirrhosis: lesson for the future? J Hepatol, 2001:34:787-8.

35 Poupon R. Ursodeoxycholic acid for primary biliary cirrhosis: lessons from the past-issues for the future. J Hepatol, 2000;32:685-8. 


\section{PostScript}

\section{BOOK REVIEW}

Aging and the Gastrointestinal Tract

Edited by A Pilotto, P Malfertheiner, P R Holt. In: Interdisciplinary Topics in Gerontology, 2003, $£ 129.75$, pp 218. ISBN 3-8055-7555-6

I became a geriatric gastroenterologist 28 years ago (to clarify any ambiguity, the term "geriatric gastroenterologist" refers to an individual interested in digestive disease in the elderly rather than an elderly person interested in digestive disease - although I will soon qualify for both). During this time a succession of slim volumes, all called something like "ageing and the gastrointestinal tract", have appeared (again to clarify, aging is the same as ageing but the latter is English spelling, the former is the way the rest of the world spell it). At first, these slim volumes were either written by gastroenterologists who knew little about elderly patients or by geriatricians who knew little about gastroenterology. I often wondered why these slim volumes appeared. I imagined them, like the collected works of minor Edwardian poets, to be a (very) minority interest. The latest of these slim volumes-Aging and the Gastrointestinal Tract, edited by Pilotto, Malfertheiner, and Holt-is a vast improvement on its predecessors. It is edited and (mostly) written by individuals who clearly know about both digestive disease and ageing. The object of the book is stated to be "to assemble.... the results of the more recent studies in geriatric gastroenterology and to review both basic research and clinical aspects of this field".

The editors have tried to get away from the normal travel down the digestive tract from mouth to anus, with side trips to the hepatobiliary system and pancreas. The first section is "a geriatric approach to gastrointestinal disorders" and contains a very good review of the epidemiology of gastrointestinal disorders in the elderly together with an interesting and fashionable chapter on "comprehensive geriatric assessment of older patients with GI disorders". The second section deals with "effect of ageing on the gastrointestinal tract", and then the conventional journey down the gut begins. This editorial idea is not entirely successful. By dividing "aging of the esophagus and stomach" from "dysphagia in the elderly" and "gastroesophageal reflux disease", there is some lack of continuity as well as repetition. The same problems apply to the liver, pancreas, and intestines.

In general, the chapters on ageing are poor, the references on gastric atrophy and physiology are largely from studies from the 1960s (now mostly superseded), apart from the author's own work. The chapter on basic ageing of the liver and pancreas is very poor. The pancreas is covered in half a page with five references. None of the exciting new ideas about the effect of age on ischaemic reperfusion injury or impaired response to many acute insults is discussed. In both "esophagus/stomach" and "liver/pancreas", the relevant clinical chapters are very much better and are exemplified by an excellent chapter on Helicobacter pylori in the elderly by two of the editors (Pilotto and Malfertheiner). This is well written, up to date, and authoritative, as well as dealing specifically with the topic of $H$ pylori in the elderly stomach.

There are some significant omissions which should perhaps have been corrected by the editors. Overall, there is little information on endoscopy in the elderly. There is also no detailed discussion of gastrointestinal bleeding-acute or occult-yet this is one of the most important problems seen in gastrointestinal disease in elderly and very elderly patients. These omissions raise the central question about this and other similar "slim volumes"-is it a specialist textbook or merely a collection of reviews which might otherwise have appeared separately in a variety of journals? If it is the former then the omissions are very significant. If it is the latter then they are perhaps not as important but it should have been made clearer that this was not intended to be a comprehensive textbook.

Finally, the editors-obviously hoping to out sell the Lord of the Rings if not Harry Potter-state, "we hope that this book will be useful for general physicians, specialists in geriatrics and gastroenterology, and all health care providers who are involved in planning the care and management of elderly people with gastrointestinal disorders". I am afraid I do not think they will become as rich as JK Rowling or even the heirs to Tolkeinrather they are destined to remain unrewarded except in heaven no doubt, like the editors of books on Edwardian poetry. The key word used by the editors above is "useful". I do not think this book is very useful to anybody over and above what one might find in the relevant chapters in the main large textbooks of geriatric medicine or gastroenterology. However, to the increasing number of aficionados, like myself, the book is interesting (as opposed to useful). I am afraid however that we aficionados are still not enough to make a fortune for either the editors or the publishers of this book.

O F W James

\section{CORRECTIONS}

In the paper by Prince et al (Gut 2004;53:865870 ), a figure in table 1 has been incorrectly labelled. The row of the table that has been printed as 'AMA titre $>1$ in $60^{\prime}$ should actually read 'AMA titre $>1$ in $160^{\prime}$. The journal apologises for this error.
In the paper by Sheil et al (Gut 2004;53:694700 ), a part of the caption to fig 1 has been printed incorrectly. The sentence currently reads "Gastrointestinal inflammatory score in the probiotic and control fed groups is shown." The sentence should have read "Gastrointestinal inflammatory score in the probiotic and control groups is shown."

\section{NOTICES}

\section{British Society of Gastroenterology Paul Brown Travel Fellowships}

The Paul Brown Travel Fellowships are awarded by the Endoscopy Committee of the BSG. They are intended to assist trainee gastroenterologists and established consultants in visits to units outside the United Kingdom for specialist experience and training in endoscopy.

Specialist registrars who have not achieved their CCST are expected to have the approval of their Postgraduate Dean and their Regional Training Director when they apply for a Travel Fellowship. Applicants are expected to provide confirmation that they have been accepted for training in the unit that they wish to visit.

Successful applicants will be expected to provide a brief written report to the Endoscopy Committee of the outcome of their visit.

Application forms are available from the British Society of Gastroenterology Office, 3 St Andrew's Place, London NWl 4LB. Email: bsg@mailbox.ulcc.ac.uk

\section{$8^{\text {th }}$ Southeast European Symposium of Paediatric Surgery}

The $8^{\text {th }}$ Southeast European Symposium of Paediatric Surgery will focus upon 'Infectious Problems in Paediatric Surgery.' The event will be held between 24-25 September 2004, at the University of Graz, Austria. For further information, please contact: Professor M E Höllwarth, Department of Paediatric Surgery, Medical University of Graz, Austria, Auenbruggerplatz 34, 8036 Graz; tel: + 43 316385 3762; fax: tel: + 43316385 3775; e-mail: kinderchirurgie@uni-graz.at.

\section{Advances in the Inflammatory Bowel Disease}

The conference for advances in the inflammatory bowel diseases will be held in Chicago, Illinois, USA on 8-9 October 2004. For further information, please contact: Coleson Chase (tel: +1 770 751 7332; fax: +1 770751 7334; e-mail: c.chase@ imedex.com; website: www.imedex.com/ calendars/therapeutic.htm). 